\title{
Monitoring Synaptic Function at the Neuromuscular Junction of a Mouse Expressing SynaptopHluorin
}

\author{
Lucia Tabares, ${ }^{1}$ Rocio Ruiz, ${ }^{1}$ Pedro Linares-Clemente, ${ }^{1}$ Michael A. Gaffield, ${ }^{3}$ Guillermo Alvarez de Toledo, ${ }^{1}$ \\ Rafael Fernandez-Chacón, ${ }^{1}$ and William J. Betz ${ }^{2}$ \\ ${ }^{1}$ Department of Medical Physiology and Biophysics, School of Medicine, University of Seville, 41009 Seville, Spain, and ${ }^{2}$ Department of Physiology and \\ Biophysics and ${ }^{3}$ Neuroscience Program, University of Colorado Medical School, Aurora, Colorado 80045
}

\begin{abstract}
We monitored presynaptic exocytosis and vesicle recycling at neuromuscular junctions of transgenic mice expressing synaptopHluorin $(\mathrm{spH})$, using simultaneous optical and electrophysiological recordings. Synaptic transmission was indistinguishable from that in wildtype controls. Fluorescence rose during and decayed monotonically after stimulus trains to the nerve, with amplitudes and decay times increasing with the amount of stimulation. The relatively large size of synaptic terminals allowed us to examine the spatial profile of fluorescence changes. We identified hot spots of exocytosis, which were stable with repeated trains. Photobleach experiments showed that $\mathrm{spH}$ freshly exposed by nerve stimulation was not preferentially retrieved by compensatory endocytosis; instead, most retrieved spH preexisted in the surface membrane. Finally, we compared fluorescence and electrical [summed end-plate potentials (EPPs)] estimates of exocytosis, which diverged during repeated trains, as fluorescence exceeded summed EPPs, although the average amplitude of miniature EPPs was unchanged. This might reflect exocytosis of spH-containing, acetylcholine-free ("empty") vesicles or other organelles during intense stimulation.
\end{abstract}

Key words: pHluorin; synaptic vesicle; FRAP; vesicle recycling; neuromuscular; synapse

\section{Introduction}

Optical measurements of exocytosis and endocytosis in nerve terminals have provided useful insights into presynaptic function. In particular, a proton-quenchable variant of green fluorescent protein (pHluorin) tagged to the lumenal domain of various synaptic vesicle proteins such as synaptobrevin [a genetic construct known as synaptopHluorin ( $\mathrm{spH})$ ] (Miesenbock et al., 1998), synaptotagmin (pHluoro-tagmin) (Fernandez-Alfonso et al., 2006), synaptophysin (sypHy) (Granseth et al., 2006), or the vesicular glutamate transporter (VGLUT-pHluorin) (Voglmaier et al., 2006), have given valuable information about the synaptic vesicle cycle, particularly in the most widely studied preparation, cultured neurons obtained from the CNS of neonatal animals transiently transfected in vitro (Sankaranarayanan and Ryan, 2000; Gandhi and Stevens, 2003; Granseth et al., 2006; Harata et al., 2006; Wienisch and Klingauf, 2006). The neuromuscular junction (NMJ), which offers additional experimental advan-

\footnotetext{
Received Feb. 14, 2007; revised April 13, 2007; accepted April 18, 2007.

This work was supported by grants from the Human Frontier Science Program Organization (RPG0045/2002-C), the European Commission for the EUSynapse Project (contract number LSHM-CT-2005-019055), Spanish Ministry of Education and Science (BFI2002-01686), the National Institutes of Health, and the Muscular Dystrophy Association of America. We thank Dr. G. Miesenbock (Yale University, New Haven, CT) for pCiNeo-VAMP-superecliptic, Drs. Weiping Han and Thomas C. Südhof (University of Texas Southwestern, Dallas, TX) for the generous gift of a modified version of Thy-1 expression vector, Pablo García-Junco Clemente for molecular engineering of constructs for transgenesis, Dr. Oscar Pintado for pronuclear injections and transgenic mice generation, Steve Fadul for unfailing assistance, and Dot Dill for expert electron microscopy. Excellent assistance was provided by Itziar Benito (mouse husbandry) and C. de Cires Segura (mouse genotyping).

Correspondence should be addressed to Lucia Tabares, Department of Medical Physiology and Biophysics, School of Medicine, University of Seville, Avenida Sanchez Pizjuan 4, 41009 Seville, Spain. E-mail: Itabares@us.es. D0I:10.1523/JNEUROSC1.0670-07.2007

Copyright $\odot 2007$ Society for Neuroscience $\quad$ 0270-6474/07/275422-09\$15.00/0
}

tages, cannot easily be cultured to maturity. To overcome this, we created a transgenic mouse (cf. Bozza et al., 2004; Li et al., 2005; Wyatt and Balice-Gordon, 2005, 2006) that expresses spH at the NMJ. In the present work, we have characterized the phenotype of this synapse, revealing several new and unexpected properties.

The NMJ possesses several useful characteristics for examining synaptic function. Compared with cultured immature neurons, the acutely dissected nerve-muscle preparation derives from an adult animal with a virtually normal phenotype. Moreover, the motor nerve terminals are relatively large compared with CNS terminals, which provides an opportunity to examine optically the spatial characteristics of vesicle exocytosis and endocytosis. Here, we report, for example, that exocytosis is not uniform along the length of motor nerve terminals. Instead, distinct regions of uncommon fluorescence increase ("hot spots") arise during nerve stimulation. Finally, at the NMJ it is relatively easy to measure electrophysiologically all of the transmitter released by the nerve, without cable decrement, because each postsynaptic muscle fiber receives only one compact synaptic input from a single neuron. We report, for example, that during a stimulus train, the summed end-plate potential (EPP) amplitudes and the fluorescence initially rise in parallel but soon diverge, summed EPPs falling below the fluorescence, despite the constancy of miniature EPP (MEPP) amplitudes.

\section{Materials and Methods}

Vector construction. The transgenic vector pThy1-SynaptopHluorin was obtained by subcloning a $1 \mathrm{~kb}$ EcoRI-SmaI fragment from pCiNeoVAMP-superecliptic (Ng et al., 2002) into the MfeI-HincII sites of a modified Thyl minigene vector that contains the Thyl genes without 
exon 3 and flanking introns to achieve neuron-specific expression (Vidal et al., 1990; Caroni, 1997; Probst et al., 2000; Han et al., 2005).

Generation of transgenic mice. Transgenic mice were generated by injection of gel-purified pThyl-SynaptopHluorin DNA digested with NotI-PvuI into fertilized oocytes using standard techniques (Hogan et al., 1994). Transgenic founders were identified by PCR. Two founders were backcrossed to FVB mice several generations before analysis. One of the lines (line A) was maintained in hemizygosis and used for experiments. The genotypes of transgenic mice were determined by PCR using the primers PLC30 (ATG AGT AAA GGA GAA GAA C) and PLC31 (TTA ACC GGT TTT GTA TAG), which amplify a 717 bp fragment contained within the coding sequence of the fluorescent protein. This mouse line has been described previously in preliminary form (Linares-Clemente et al., 2005).

Acute experimental conditions. The levator auris nerve-muscle preparation was dissected as described previously (Katz et al., 1996) and pinned in a Sylgard-lined chamber, and the nerve was drawn into a suction-stimulating electrode. The preparation was perfused continuously with a solution gassed with $95 \% \mathrm{O}_{2} / 5 \% \mathrm{CO}_{2}$ containing the following (in mM): $125 \mathrm{NaCl}, 5 \mathrm{KCl}, 2 \mathrm{CaCl}_{2}, 1 \mathrm{MgCl}_{2}, 25 \mathrm{NaHCO}_{3}$, and 15 glucose. In some experiments, the calcium-ion concentration was increased to $5 \mathrm{~mm}$ (with a corresponding reduction of $\mathrm{NaCl}$ ). Muscle twitches were blocked with curare (1.5-2 $\mu \mathrm{M}$ ) or $\mu$-conotoxin GIIIB (1 $\mu \mathrm{M}$; Alomone Labs, Jerusalem, Israel). All experiments were performed at room temperature. Stimulus trains were delivered at $100 \mathrm{~Hz}$, a frequency at which fast twitch muscles are known to operate (Hennig and Lomo, 1985), although only for brief periods, so that trains lasting longer than $\sim 0.5 \mathrm{~s}$ were probably not physiological. Lower frequencies were also used, with similar results (data not shown). For most experiments, an Olympus (Tokyo, Japan) BX50WI upright epifluorescence microscope fitted with a water-immersion LUMPlanFI $60 \times / 0.90$ (Olympus) was used, with images acquired with a Hamamatsu (Hamamatsu, Japan) Orca ER or 9100-02 camera. Fluorescence responses were taken every 0.1-1 s using time-lapse routines generated by the camera software (Wasabi, Hamamatsu, Japan). For the photobleach and fluorescence recovery after photobleaching (FRAP) experiments in Figure 4, we used a Zeiss (Thornwood, NY) 510 laser-scanning confocal microscope (Gaffield et al., 2006). Electrophysiological recordings were made with intracellular micropipettes filled with $3 \mathrm{M} \mathrm{KCl}(10-15 \mathrm{M} \Omega)$, and data were acquired with Chart software (ADInstruments, Castle Hill, New South Wales, Australia) on a computer running Windows XP.

Image analysis. Image analysis was performed with custom routines written in Matlab. Typically, images in a time-lapse series were first aligned using a least-squares fit to a region of interest (ROI), denoised using singular value decomposition (Prechtl et al., 1997), and background subtracted using an ROI near the nerve terminal. ROIs of the terminal were selected by picking square regions, by drawing regions, or automatically with brightness thresholding. In some cases, ROIs were determined from low-pass-filtered images (two-dimensional moving bin average of five to seven pixels with Gaussian weighting using an SD of $0.65-1$ pixel), with actual measurements made on the original images. For determining endocytic rate on a pixel-by-pixel basis (see Fig. $3 D$ ), we fitted the decay time course for each pixel position, using a linear best-fit model.

\section{Results}

The hemizygous spH-expressing mice appear indistinguishable from their wild-type littermates and reproduce normally. They exhibit no evident muscle weakness or lack of coordination. Electrophysiological recordings of action potentials and synaptic potentials from postsynaptic muscle fibers are likewise indistinguishable from wild type, as are visual observations of muscle twitches and tetani. Electron microscopy of motor nerve terminals revealed no evident differences from controls (results not shown).

A typical imaging experiment is shown in Figure 1. The levator auris nerve-muscle preparation was pinned in a recording chamber and superfused continuously with normal Ringer's solution
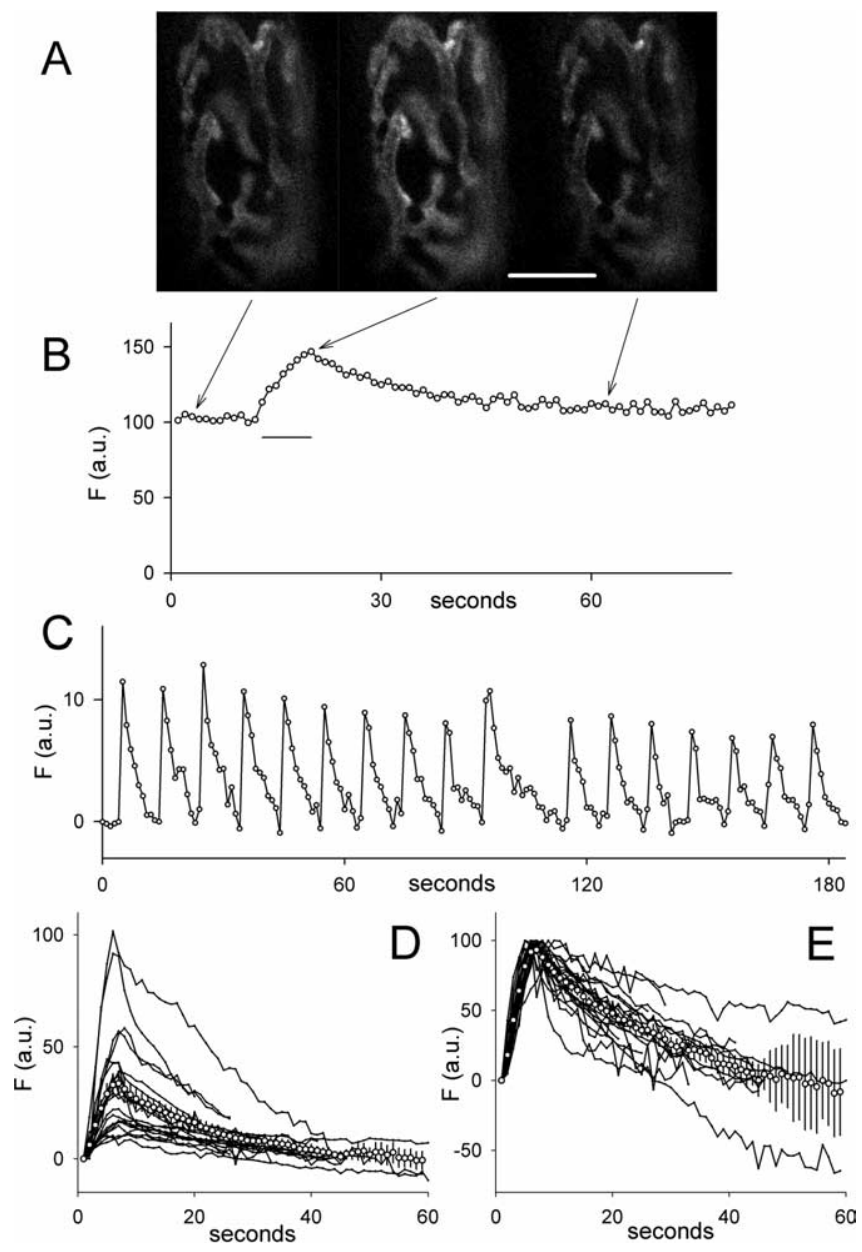

Figure 1. SynaptopHluorin fluorescence reliably reflects nerve stimulation. $\boldsymbol{A}$, Transgenic mouse motor nerve terminal expressing spH before stimulation (left), at the end of a stimulus train ( $100 \mathrm{~Hz}$ for $8 \mathrm{~s}$; middle), and $1 \mathrm{~min}$ after the train ended (right). Images were background subtracted. Scale bar, $10 \mu \mathrm{m}$. $\boldsymbol{B}$, The average fluorescence $(F)$ of the terminal, which increased $\sim 50 \%$ during the train (horizontal bar) and then decayed exponentially with a time constant of $14 \mathrm{~s}$. C, The robust reliability of the fluorescence rise during repeated trains (each $100 \mathrm{~Hz}$ for $0.5 \mathrm{~s}$ ) is clearly evident. Successive peak amplitudes decayed by $\sim 3 \%$. D, Fluorescence of 23 different terminals (background subtracted), offset (no scaling) to equal zero immediately before the train. Open circles show mean \pm SEM. $\boldsymbol{E}$, Same data as $\boldsymbol{D}$, normalized to peak $F$ values to show variation in recovery after the stimulus train ended. The best-fit exponential (open circles) has a decay time constant of $27 \mathrm{~s}\left(r^{2}=0.996\right)$. a.u., Arbitrary units.

at room temperature. Motor nerve terminals were identified with bright-field optics and then imaged with conventional epifluorescence microscopy and a CCD camera (see Materials and Methods). The three panels in Figure $1 A$ show the terminal before, during, and after tetanic stimulation of the nerve $(100 \mathrm{~Hz}$ for $10 \mathrm{~s}$ ). Surface membrane $\mathrm{spH}$ produced most of the initial fluorescence visible in the left panel (for details, see supplemental Fig. S2, available at www.jneurosci.org as supplemental material). The fluorescence increased during stimulation (middle) and then grew dim again after the stimulation ended (right). To quantify the results, images were aligned and background subtracted, terminal branches were outlined, and the average fluorescence in each image was measured (Fig. $1 B$ ). Nerve stimulation was delivered during the time marked by the horizontal line. The fluorescence decay, representing the sequential processes of endocytosis of $\mathrm{spH}$ and its quenching by acidification, could be fitted with a single exponential (time constant $=14 \mathrm{~s} ; r^{2}=0.94$ ). The responses were remarkably reproducible. Figure $1 C$ shows 17 con- 
secutive stimulus trains (each $100 \mathrm{~Hz}$ for $0.5 \mathrm{~s}$ ). The average peak response declined $\sim 3 \%$ per train, probably because of photobleaching. Responses (both increases and decay time constants) between different terminals were less consistent than in a single terminal. Figure $1 D$ shows responses from 23 different terminals, each responding to an identical stimulus train (100 Hz for $5 \mathrm{~s})$. There is nearly an order of magnitude range in peak fluorescence increase. The fluorescence decay rates also varied, as shown in Figure $1 E$, in which the same data are normalized to peak fluorescence (average time constant of fluorescence decay $=27 \mathrm{~s} ; r^{2}$ $=0.995)$.

We performed a number of different experiments confirming that the NMJ performed fundamentally like other preparations transfected with spH (supplemental Figs. S1, S2, available at www. jneurosci.org as supplemental material). For example, both the magnitude of the fluorescence increase and the rate of decay after stimulation depended on the amount of stimulation, with longer stimulus trains producing larger fluorescence rises and slower decays (supplemental Fig. S1, available at www.jneurosci.org as supplemental material). The total intracellular $\mathrm{spH}$ pool was probed by alkalinizing the intracellular (including intravesicular) fluids by exposing the preparation to $\mathrm{NH}_{4} \mathrm{Cl}$. The fluorescence intensity typically rose 5-10 times more than was produced by nerve stimulation, indicating a relatively large "reserve pool" of intracellular vesicles (supplemental Fig. S2 A, available at www. jneurosci.org as supplemental material). Resting terminals showed some fluorescence; we estimate that at least two-thirds of this background signal arose from surface $\mathrm{spH}$; most of the rest arose from autofluorescence, with a small contribution from unquenched intracellular $\mathrm{spH}$ (supplemental Fig. S2 B, available at www.jneurosci.org as supplemental material). Finally, we showed that folimycin, an inhibitor of the vesicular proton pump, greatly reduced the rate of fluorescence decay after nerve stimulation, as expected, and also reduced the amplitude of fluorescence changes with repeated stimulus trains, suggesting that recycled vesicles were reused (supplemental Fig. S2C, available at www.jneurosci.org as supplemental material).

\section{Spatial analysis}

Mouse motor nerve terminals are large enough $(2-3 \mu \mathrm{m}$ in diameter and several tens of micrometers in length) to permit detailed spatial analysis. We monitored fluorescence changes in different regions of the same terminal and asked whether the amplitudes and time courses of fluorescence changes were spatially uniform. Analysis of a typical experiment is shown in Figure 2. The average fluorescence of the entire terminal is plotted in Figure $2 A$; the inset shows an image of the resting terminal. We measured on a pixel-by-pixel basis the difference between baseline and peak response produced by four trains of tetanic stimulation (three control frames immediately preceding the stimulus were averaged and subtracted from the average of three frames at the peak). Each panel in Figure $2 B$ shows a pseudocolored difference image (peak minus baseline) for one of the stimulus trains. Considering only the top panel, it is clear that the responses were not uniform but that distinct areas (red) gave a much greater response than other areas (blue). To test whether these apparent hot spots were real, we repeated the trains (Fig. $2 B$, bottom three panels) and found that the hot spots reappeared in the same locations. This impression is quantified in Figure 2C. Here, for each panel we compared individual pixel locations for each stimulus train. The red lines show the individual values of the fifty pixels that, on average, showed the largest fluorescence increases during the trains. A similar process was performed for the pixels that were 500-550 in rank order of average fluorescence increase (green), and for 3000-3050 (blue). It is clear that between colors there is virtually no overlap in the pixel values. Another test (Fig. $2 D)$ shows for each pixel location the mean fluorescence rise for the four trains ( $x$-axis) plotted against the variance ( $y$-axis). The linear relation $\left(r^{2}=0.25\right)$ again suggests a reasonably consistent response from trial to trial. In other words, knowing a pixel value for one train predicted well its value for all other trains, leading to the conclusion that the hot spots are real and stable over time. Finally, we showed that the initial level of background fluorescence intensity was a poor predictor of hot spot locations. For example, the image in Figure $2 \mathrm{~A}$ (inset) shows the resting terminal, with a large, bright spot near the middle, but no hot spot appeared there. The fluorescence of this spot is plotted in Figure $2 E$ (top trace); two other regions, initially much dimmer, are plotted, and show clear exocytic responses to nerve stimulation. Figure $2 \mathrm{~F}$ shows on a pixel-by-pixel basis that no significant correlation existed $\left(r^{2}=0.02\right)$ between initial fluorescence and response to stimulation.

We next asked whether the rate of fluorescence decay (reflecting endocytosis and requenching by acidification) correlated with hot spot locations. Figure $3 A$ shows a typical terminal. A stimulus train lasting $2 \mathrm{~s}$ produced a clear rise in average fluorescence, followed by a smooth decay (Fig. 3B). Exocytic hot spots were clearly detectable (Fig. $3 C$ ). We then measured the rate of decay of fluorescence at all 5560 pixels in the terminal region [for each pixel position, we used a linear curve fit between frame 17 (the peak fluorescence change) and the last frame $(51 ; 1 \mathrm{~Hz}$ imaging rate)]. The results are shown in Figure $3 D$, in which red regions indicate fast decay of fluorescence, and blue regions indicate slow decay of fluorescence. Figure $3 E$ shows the correlation coefficient $\left(r^{2}\right)$ for the curve fit at each pixel location; it is clear that throughout most of the terminal the fitting procedure was acceptable. Visual inspection of hot spots (Fig. 3C) and decay rates (Fig. $3 D$ ) shows a reasonable, although not perfect, alignment between the two, suggesting that exocytic hot spots are also at or near sites of relatively rapid endocytosis and quenching of $\mathrm{spH}$ fluorescence. This is quantified in Figure $3 F$, in which the pixel values in $C$ and $D$ are plotted against each other $\left(r^{2}=0.59\right)$.

\section{Photobleaching experiments}

We performed additional imaging with a Zeiss 510 microscope equipped for laser photobleaching, modifying an experimental paradigm performed on cultured hippocampal synapses (Fernandez-Alfonso et al., 2006; Wienisch and Klingauf, 2006) to distinguish between retrieval of $\mathrm{spH}$ preexisting on the cell surface and $\mathrm{spH}$ freshly exocytosed during the stimulus train. The relatively large size of the NMJ offered a useful advantage, namely that it was relatively easy to bleach only part of the terminal, leaving the remainder to serve as a control (cf. Wyatt and BaliceGordon, 2006). Figure $4 A$ shows a terminal immediately before (top) and after (bottom) the right half of it was bleached. We performed three types of bleaching experiments, involving one, two, or three sequential stimulus trains (we waited 5-20 min between trains to insure full recovery from synaptic depression). First, as shown in Figure $4 B$, we compared responses to a single stimulus train (horizontal bar) in bleached regions (open circles) and control (nonbleached) regions (filled circles). The delay between the bleach and the start of stimulation allowed us to measure the FRAP rate of the $\mathrm{spH}$ that preexisted in the surface membrane; the solid line preceding the start of stimulation is a best-fit single-exponential curve $(\tau=2.1 \mathrm{~s}$; mobile fraction, $16.2 \%)$. Subsequent nerve stimulation caused approximately equal rises 

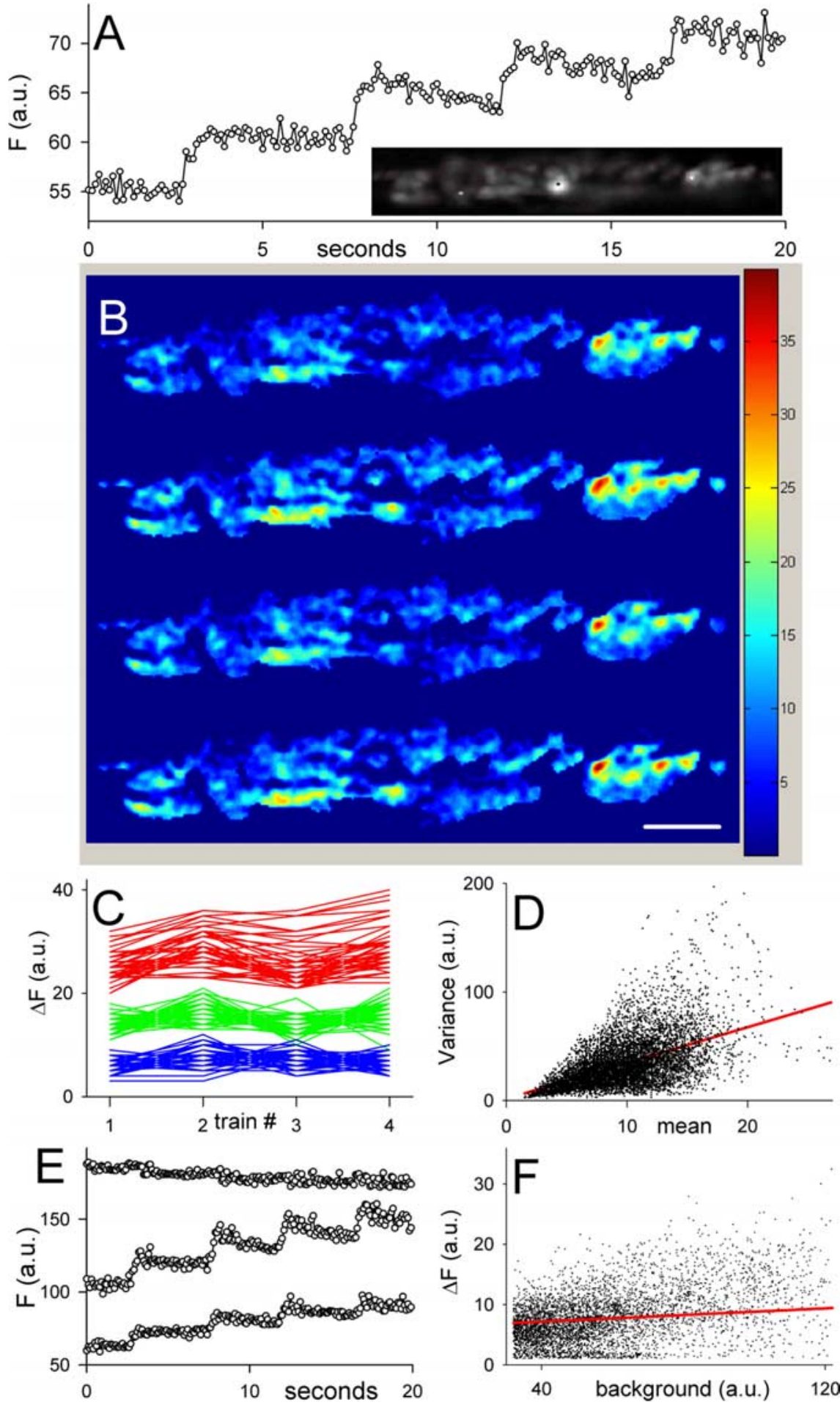

Figure 2. Spatial analysis of exocytic hot spots. $\boldsymbol{A}$, Fluorescence responses (averaged over the entire terminal) to four stimulus trains (each $100 \mathrm{~Hz}$ for $0.5 \mathrm{~s}$ ). Inset, Image of resting terminal. The bright spot near the center ${ }^{*}$ ) showed no fluorescence increase during nerve stimulation. $\boldsymbol{B}$, Fluorescence increases (difference between average of last 3 control images before train and average of 3 images at the peak of fluorescence) are shown for each of the four trains on a pixel-by-pixel basis. Regions of large increases (red) tended to recur at the same positions for each train. Scale bar, $10 \mu \mathrm{m}$. $\boldsymbol{C}$, The images from $\boldsymbol{B}$ were aligned, and pixels at the same positions within the boundaries of the terminal ( $n=3806$ pixels) were averaged. The 50 positions with the highest average fluorescence increase were identified, and the individual pixel values from each train were plotted (red). The same procedure was followed for the positions with lowest (blue) and middle (green) rises in fluorescence. The graph shows that pixel values in one train predict pixel values in all trains. $\boldsymbol{D}$, The mean-variance scatter plot for each pixel position shows a reasonably strong correlation $\left(r^{2}=0.59\right)$, again suggesting consistent responses from train to train. $\boldsymbol{E}$, The fluorescence of three spots are plotted. The spot with the highest fluorescence at the outset $\left({ }^{*}\right.$ in $\boldsymbol{A}$ ) showed no response at all during stimulation, whereas the much dimmer spots showed robust responses. $\boldsymbol{F}$, Pixel-by-pixel scatter plot of initial background fluorescence versus rise in fluorescence during stimulation shows virtually no correlation $\left(r^{2}=0.02\right)$. a.u., Arbitrary units. in fluorescence in both bleached and control regions, but the recovery that followed was approximately fivefold slower in the bleached region. This suggests that under these conditions, endocytosis retrieves mainly $\mathrm{spH}$ that preexisted on the surface, rather than that exposed during the train.

The next two experiments served primarily as controls to check for signs of photodamage in bleached regions. Figure $4 C$ shows that exocytosis was not impaired by bleaching, by examining responses in the same region to two consecutive trains, with photobleaching of the region between the trains. The top two traces show responses to long trains $(30 \mathrm{~s})$, and the bottom two traces show responses to short (5 s) trains, all at $100 \mathrm{~Hz}$. Photobleached traces have been offset vertically (without scaling) to superimpose fluorescence values immediately before nerve stimulation. The amplitude of the stimulus-induced rise in fluorescence after bleaching is similar to that in controls (although the recovery was slower, as in Fig. 4B). Figure $4 D$ shows that endocytosis was not impaired by photobleaching. For this, we gave three consecutive trains and measured responses to each train in the same region. Bleaching occurred after the first train. In Figure $4 D$, we show only the first and third responses (the second response, not shown, occurred immediately after the bleaching, and resembled that in Fig. $4 B$, being of normal amplitude but slowed recovery). Filled circles in Fig. $4 D$ show the first response (control), and open circles show the last response (second train after bleaching). Results are shown for both $30 \mathrm{~s}$ trains (top pair) and $5 \mathrm{~s}$ trains (bottom pair). The smooth lines show best singleexponential fits to the fluorescence decay; the time constants were similar [27 and $31 \mathrm{~s}$ for control (top) and third train (bottom); $r^{2}=0.95$ and 0.83 , respectively], showing that the endocytic and $\mathrm{spH}$ quenching machinery were intact after the bleach. The fact that the third response was smaller than control suggests that vesicles retrieved after the first postbleach train (which contained a significant amount of bleached $\mathrm{spH}$ ) recycled and were "reused" in the last train.

\section{Combined fluorescence \\ and electrophysiology}

Because mammalian skeletal muscle fibers are innervated by a single neuron, at a single location, it is possible to monitor simultaneously $\mathrm{spH}$ fluorescence and all postsynaptic electrical signals, without cable decrement, and compare directly estimates of exocytosis obtained by the two 
methods. The cumulative sum of EPPs [corrected for voltage saturation (nonlinear summation)] should be proportional to the rise in fluorescence (corrected for requenching). Figure $5 A$ shows that a $50 \mathrm{~s}$ stimulus train $(100 \mathrm{~Hz})$ caused a large rise in fluorescence. EPPs recorded during the train are shown in Figure $5 B$ (insets show responses on a faster time scale). EPP amplitudes were corrected for voltage saturation (assuming -10 or $0 \mathrm{mV}$ as reversal potential) and then summed, giving the lines ( $\mathrm{E}$ EPP) in Figure $5 C$ (top line, -10 $\mathrm{mV}$ reversal potential). Fluorescence values were corrected (increased) for loss of fluorescence during stimulation (caused by endocytosis and acid requenching) by assuming a rate equal to that observed after the train ended. The endocytic rate may have been faster than this, especially early in the train, which would lead to an undercorrection for fluorescence loss. The two curves (filled circles) show the results [uncorrected (bottom) and corrected (top)]. Note that the fluorescence and summed EPP values are plotted on separate $y$-axes, scaled (linearly) to superimpose the data at early times. This is equivalent to assuming that at the beginning of the train, fluorescence and summed EPPs do measure the same thing. It is clear, however, that beginning $\sim 20 \mathrm{~s}$ after the start of the stimulus train, the two curves deviate significantly, with the summed EPPs falling below the fluorescence. An alternative scaling method is shown in Figure $5 D$. Here, the values were made equal at the end of the stimulus train (again by a single scaling factor); for most of the time, the fluorescence curve $(\Delta F)$ now lies below the summed EPP curve. We could correct (boost) the fluorescence curve by assuming that requenching (endocytosis plus acidification) was very fast initially and slowed down during the train. Specifically, if we assumed that initially the rate of fluorescence disappearance was $\sim 90 \%$ per second and then progressively slowed during the train, as shown in Figure $5 E$, we could make the two curves in Figure $5 D$ virtually superimpose. This very fast requenching is improbably high, given estimates of the amount and kinetics of kiss-and-run exocytosis (Harata et al., 2006; He et al., 2006), and the measured rate of requenching after endocytosis (Atluri and Ryan, 2006), and we performed subsequent analyses using the first technique (i.e., aligning the two curves at early times). We also examined the effect using short, repeated trains, with similar results (Fig. 5, compare $F, C$ ). In this example, each vertical jump corresponds to a brief, $100 \mathrm{~Hz}$ stimulus train. Results from this and four other experiments are shown in Figure $5 G$. Here, for each train, the ratio of summed EPPs to fluorescence change is plotted, with the ratio normalized to 1.0 for the first train in each experiment. The duration of each train is given by the width of the symbol $(2,4,10$, or $16 \mathrm{~s})$. In every case, the ratio decreases with successive trains, showing that the transmitter release detected by the intracellular recording fell further and further behind the fluorescence rise. We did not test extensively for the reversibility of the phenomenon, but we observed that after a rest of $\sim 10 \mathrm{~min}$, the fluorescence-summed
EPP discrepancy had mostly or completely disappeared (results not shown).

Several testable explanations for the discrepancy in fluorescence and electrical recordings are immediately evident. If postsynaptic receptors were desensitized during tetanic stimulation, if recycling vesicles were only partially refilled with acetylcholine before undergoing repeated exocytosis, or if muscle fiber input resistance fell, then the EPPs would decrease in amplitude with no commensurate decrease in fluorescence. To test for these possibilities, we made recordings from muscle fibers in which MEPPs were clearly visible (Fig. 6). Muscle contractions were blocked with $\mu$-conotoxin, which preserved EPP and MEPP amplitudes and allowed us to monitor MEPP frequency and amplitude continuously and also to calculate EPP quantum content directly as the ratio of EPP amplitude to mean MEPP amplitude. MEPPs were recorded continuously before, during, and after stimulus trains. Typical recordings are shown in Figure $6 A$. These were obtained at the times marked by arrows in Figure $6 B$, which plots MEPP frequency (averaged over $1 \mathrm{~s}$ intervals) during the entire experiment. Figure $6 C$ shows MEPP amplitudes (moving bin average of 9) during the entire experiment, and Figure $6 D$ shows the averages of all MEPPs between each train. Average MEPP amplitudes during and after each train varied by no more than 4\%. Peak amplitudes of evoked EPPs were also recorded, corrected for voltage saturation, and converted to number of quanta. Figure $6 \mathrm{E}$ shows the cumulative release of all quanta, both individual MEPPs and evoked EPPs, and their sum. By the end of the experiment, approximately one-half of the released quanta had arisen from evoked EPPs and one-half from asynchronous release. We sought to identify every MEPP that occurred during each train. Figure $6 F$ shows recordings obtained 

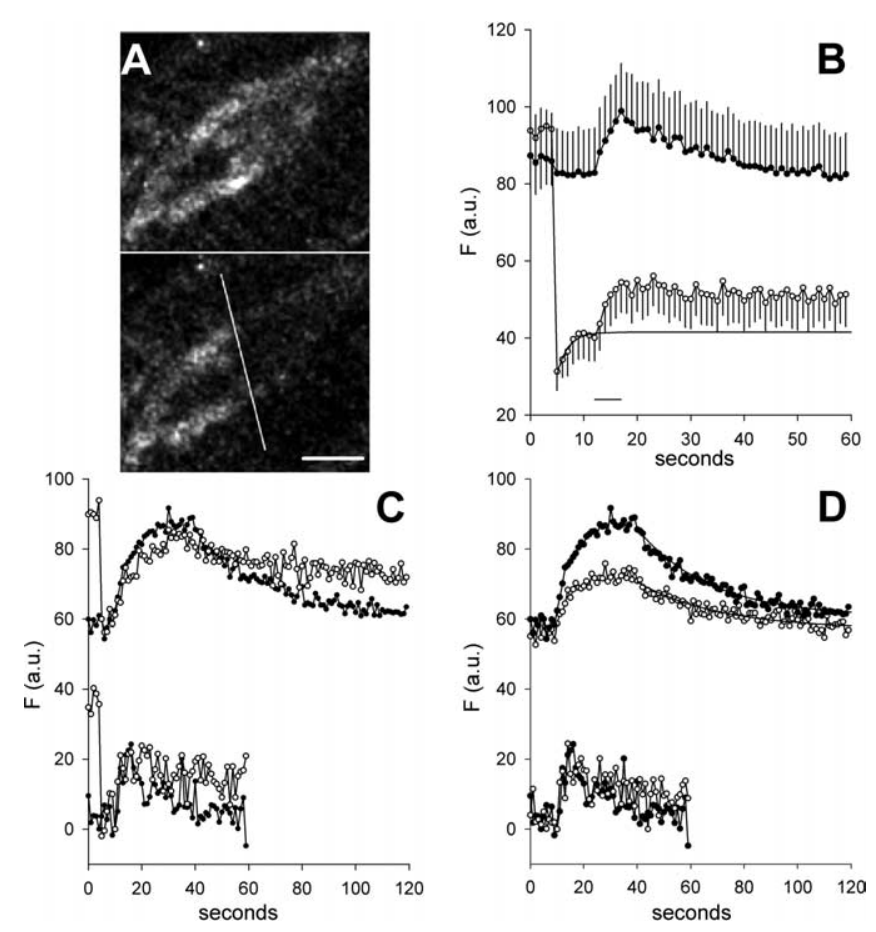

Figure 4. $\mathrm{spH}$ newly exposed by nerve stimulation is not recycled in preference to $\mathrm{spH}$ preexisting on the surface. $\boldsymbol{A}$, Fluorescence images of terminal before (top) and after (bottom) a bleach of surface $\mathrm{spH}$. The terminal area to the right of the white line was bleached. Scale bar, 10 $\mu \mathrm{m}$. $\boldsymbol{B}$, One stimulus train. Average \pm SEM fluorescence of two regions, one control (nonbleached; filled circles) and one bleached (open circles). After the bleach (given at $t=4 \mathrm{~s}$ ), but before nerve stimulation started (at $t=12 \mathrm{~s}$ ), surface fluorescence recovered with a time constant of $2.1 \mathrm{~s}$ (solid line). Nerve stimulation ( $100 \mathrm{~Hz}$ for $5 \mathrm{~s}$; horizontal bar) caused approximately equal increases in fluorescence in both control and bleached regions, but the fluorescence decay after stimulation ended was much slower in the bleached region: best-fit singleexponential time constants were $20 \mathrm{~s}$ (control) and $112 \mathrm{~s}$ (bleached); expressed alternatively, $30 \mathrm{~s}$ after the end of the stimulus train, the amount of recovery was $100 \%$ (control) and $22 \%$ (bleached). The solid line shows background (surface) fluorescence. Data are from nine terminals in three muscles. $\boldsymbol{C}$, Bleaching did not inhibit exocytosis. Two stimulus trains were given; part of the terminal was bleached shortly before the second train. $\mathrm{spH}$ signals were measured from the same region before (filled circles) and after (open circles) bleaching of that region. Stimulus trains were $100 \mathrm{~Hz}$ for $30 \mathrm{~s}$ (top pair) or $5 \mathrm{~s}$ (bottom pair), separated by 20 or $5 \mathrm{~min}$, respectively. In both cases, the amplitudes of the fluorescence increases were nearly identical for control and postbleach trains. The decay rate of fluorescence was slower after the bleach, as in $\boldsymbol{A}$. For each postbleach response, a constant was added (no scaling) so that traces superimposed immediately before each train. $\boldsymbol{D}$, Bleaching did not inhibit requenching. The protocol was like $\boldsymbol{C}$, with an additional (third) stimulus train given after a rest period. Filled circles show control (prebleach) responses. Open circles show responses to the last train (responses to the second train are not shown). The fluorescence decay rates were not significantly different: best-fit single-exponential time constants (smooth lines) were $27 \mathrm{~s}$ for control (top) and $31 \mathrm{~s}$ for third train (bottom; $r^{2}=0.95$ and 0.83 , respectively), showing that bleaching did not interfere with requenching. In addition, the exocytic response amplitude was significantly reduced after bleaching in the top pair ( 30 s train), suggesting that released vesicles contained bleached spH that was retrieved after the second train and trafficked back to the recycling pool. In $C$ and $D, 30 \mathrm{~s}$ stimulation traces are typical results, whereas $5 \mathrm{~s}$ traces are averages of at least two experiments. a.u., Arbitrary units.

during the early, middle, and late part of train 6 (taken at times marked by arrows in Fig. 6G, middle). By the end of the train (lowest trace), evoked EPPs had greatly declined, and MEPPs, which were easily detected, had increased greatly in frequency. Of the 1536 quanta released during train 6, 243 came from asynchronous MEPPs. We wondered whether undetected MEPPs could have explained the discrepancy between quanta and fluorescence. In fact, an additional 1832 MEPPs would be required to erase the discrepancy. It seems extremely unlikely that we would have missed so many asynchronous quanta in our measurements, particularly given the favorable signal-to-noise ratio of the recordings (Fig. $6 F$ ). Figure $6 G$ shows the rundown of EPP amplitudes during three of the trains; arrows in the middle panel point to recordings shown on an expanded time scale in Figure $6 F$. Figure $6 \mathrm{H}$ shows the observed fluorescence, averaged over the entire terminal (the drop at the beginning of each train was attributable to slight movement). Imaging was halted after six trains, whereas electrical recordings continued for three more trains. Figure $6 I$ shows the fluorescence increase for each train (filled circles) and the total quanta released for each train (large open circles), scaled linearly on separate axes to superimpose for the first train. As in Figures 4 and 5, the summed EPP data fell progressively below the fluorescence responses, which remained nearly constant.

\section{Discussion}

The neuromuscular junction of the mouse expressing $\mathrm{spH}$ has given results that confirm some aspects of synaptic function reported in cultured neonatal CNS neurons transiently transfected with $\mathrm{spH}$ and that reveal new information not reported previously, because of the relatively large size and easy acquisition of combined electrical and optical data at the NMJ. At individual NMJs, the consistency of responses to repeated stimuli (Fig. 1C) is remarkable and greatly facilitates experiments, compared, for example, with the use of FM dyes, which must be reloaded for each stimulus train. A number of experiments gave results consistent with work on other preparations transfected with spH. For example, the fluorescence intensity and subsequent time constant of decay both increased with increasing numbers of stimuli, the relatively large fluorescence increase in response to $\mathrm{NH}_{4} \mathrm{Cl}$ application suggests that the recycling pool of synaptic vesicles is relatively small, $\sim 20 \%$ of the total $\mathrm{spH}$ is in the surface membrane at rest, and the inhibition by folimycin of $\mathrm{spH}$ requenching after a stimulus train are all consistent with work on other preparations (see supplemental material, available at www.jneurosci.org).

The relatively large size of the nerve terminals allowed us to examine the spatial properties of exocytosis. Exocytic hot spots were evident, and they reappeared in the same positions with repeated stimulus trains (Fig. 2). The scattered locations of exocytic sites have been most clearly observed functionally in retinal bipolar neurons (Zenisek et al., 2000). In mouse motor nerve terminals, immunostaining of active zones reveals a similar randomly distributed array of diffraction-limited spots separated by submicron distances (Nishimune et al., 2004). The hot spots that we observed were not so distinctly punctate and covered an area that would encompass several active zones. The relatively intense stimulation that we used, which produced exocytosis of thousands of vesicles, may have obscured such an underlying punctate pattern. Our hot spots are more reminiscent of those detected with focal electrical (Zefirov et al., 1995) or optical (Wu and Betz, 1999) recordings at the frog NMJ. They may reflect some other process, such as accumulations of synaptic vesicles or calcium channels. The initial (prestimulus) fluorescence level, which primarily reflects $\mathrm{spH}$ stranded in the surface, was not a predictor of the exocytic response to stimulation, either in the amount of fluorescence increase or in the rate of compensatory endocytosis (Fig. 2F).

The interpretation of the fluorescence decay rate, which paralleled with reasonable accuracy exocytic hot spot locations, is more complex. Loss of $\mathrm{spH}$ fluorescence at any single location could reflect endocytosis and requenching or lateral movement of spH in the surface membrane (Granseth et al., 
2006). A complete analysis will require additional experiments, using, for example, FRAP and rapid acid superfusion. In any case, we did not observe, for example, wholesale accumulation of $\mathrm{spH}$ at the lateral margins of the terminals, where endocytosis is prominent after intense stimulation at the frog NMJ (Heuser and Reese, 1973). Thus, although increasing stimulation slowed endocytosis across the entire terminal (Fig. 2), within any region the rate of requenching was adaptively matched to exocytosis.

The nature of lateral diffusion of $\mathrm{spH}$ in the surface membrane is a subject of considerable interest. Heuser and Reese (1973) suggested that during intense stimulation, vesicle components move in the surface membrane to the lateral margins of the terminal before undergoing endocytosis. In snake neuromuscular boutons, "endocytic active zones" were identified immediately adjacent to exocytic active zones (Teng et al., 1999). More recent studies on cultured hippocampal neurons have concluded that compensatory endocytosis retrieves membrane that is different from that recently added by exocytosis (FernandezAlfonso et al., 2006; Wienisch and Klingauf, 2006). We performed similar photobleach experiments, with similar results, with the added advantage that we could directly compare bleached and nonbleached regions within the same terminal, a useful control. After part of a nerve terminal was bleached, subsequent nerve stimulation produced a normal rise in fluorescence as new $\mathrm{spH}$ was externalized, but the subsequent decay of fluorescence was slowed approximately fivefold (Fig. 4B), consistent with the idea that compensatory endocytosis after intense stimulation does not retrieve preferentially $\mathrm{spH}$ molecules that were freshly inserted in the surface membrane. Other photobleach experiments showed that neither exocytosis nor endocytosis was inhibited by the bleaching and that vesicles containing bleached $\mathrm{spH}$ were reused with subsequent stimulus trains.

The NMJ offers an opportunity to combine with unique precision optical and electrical measurements at single synapses. Synaptic potentials originate at a single synapse, with little or no cable decrement between it and the recording electrode, and optical signals arise entirely from a single compact end plate. We recorded evoked EPPs and $\mathrm{spH}$ fluorescence simultaneously, each of which should provide a reliable measure of exocytosis and transmitter secretion. We found, however, that the two measures reliably diverged, with summed EPPs lagging behind fluorescence during repetitive stimulation. Because average MEPP amplitude remained constant, it seems reasonable to discount, if not rule out entirely, postsynaptic desensitization, acetylcholine
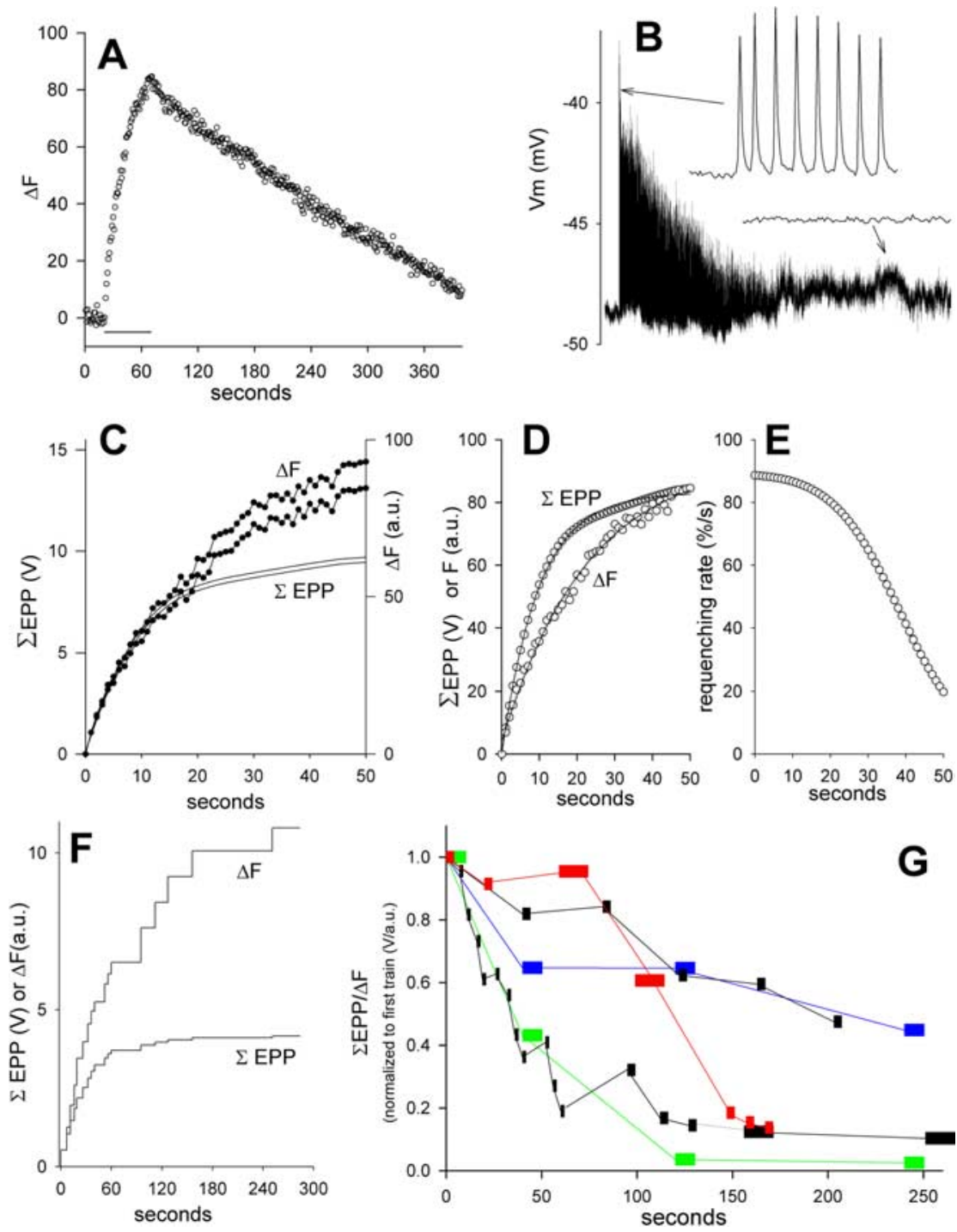

Figure 5. Combined electrophysiological and fluorescence estimates of transmitter secretion during prolonged stimulation do not agree. $\boldsymbol{A}$, Fluorescence changes during a 50 s stimulus train $(100 \mathrm{~Hz}$; marked by horizontal line). $\boldsymbol{B}$, EPPs recorded during the train. Insets, Sample EPPs (at times marked by arrows) at expanded time scale (10 ms between successive EPPs). During the train, EPPs declined and disappeared into the recording noise. C, Summed EPPs (smooth lines) and fluorescence (filled circles) during the stimulus train, plotted on separate axes (both linear, starting at zero). EPP amplitudes were summed after correction for nonlinear voltage summation using reversal potentials of $0 \mathrm{mV}$ (bottom smooth line) or $-10 \mathrm{mV}$ (top smooth line). Raw fluorescence change (bottom filled circle trace) was corrected for endocytosis and reacidification (top filled circle trace), using a fluorescence decay rate (0.2 a.u./s) obtained from a linear fit to the observed fluorescence decay after the end of stimulation $(\boldsymbol{A})$. The EPP and fluorescence curves superimpose well at early times but deviate significantly after 15-20 s of stimulation, with summed EPPs falling below the fluorescence. $\boldsymbol{D}$, An alternative scaling method, in which the last rather than the first points in the summed EPP and fluorescence curves were superimposed with a single scaling factor. Now, the fluorescence falls below the summed EPP curve. The curves were each fitted by single rising exponentials (smooth lines). The fluorescence curve was then increased point by point to equal the summed EPP curve, by assuming that some fluorescence escaped full detection because of rapid endocytosis and requenching. $\boldsymbol{E}$, Percentage of total exocytosis that would be required to be requenched in one second to explain the discrepancy between the two curves. The high amount required makes this an unlikely explanation. $\boldsymbol{F}$, The fluorescence- $\Sigma$ EPP disparity persisted with multiple short trains (each marked by an upward jump in the data). The fluorescence increases (top trace; no correction for requenching) and summed EPPs (bottom trace, assuming $-10 \mathrm{mV}$ reversal potential) produced by each train were each added and scaled to superimpose after the first train. Subsequently and progressively, the EPP curve fell below the fluorescence curve. Train durations were $2 \mathrm{~s}$ (first 12), 4 s (next 3), and 10 s (last 2). G, Results from $\boldsymbol{F}$ (bottom black trace) and four other experiments, in which the ratio of summed EPPs to fluorescence change are plotted for each train, normalized to a value of 1.0 for the first train. Different colors are used for clarity. The duration of each train is equal to the width of the symbol. Trains were 2, 4, 10, or $16 \mathrm{~s}$ in duration. EPPs were corrected for nonlinear voltage summation assuming a reversal potential of $-10 \mathrm{mV}$; fluorescence changes were corrected for endocytosis as in C. A clear decrease in the ratio is evident in every experiment. a.u., Arbitrary units. 

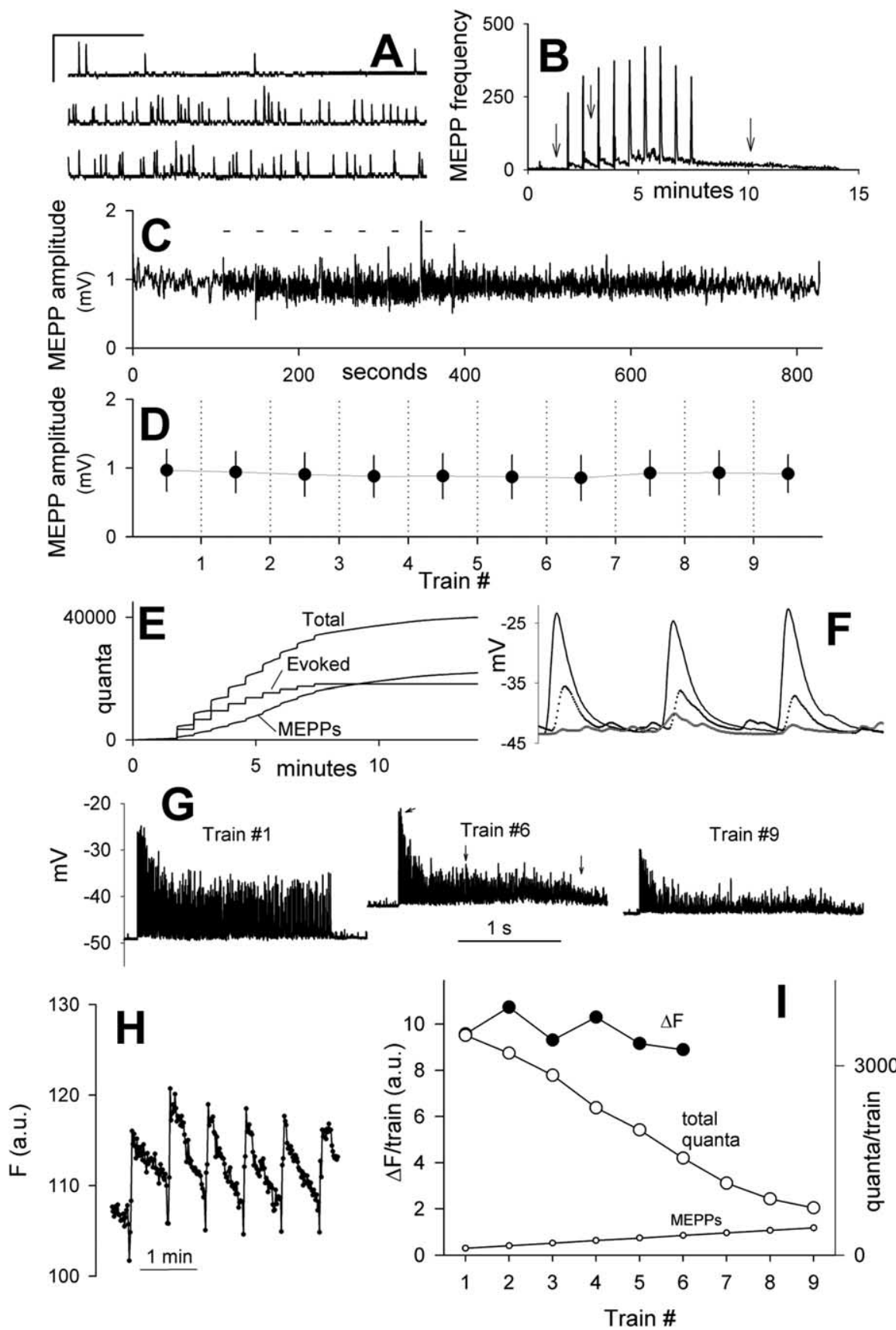

Figure 6. The fluorescence- $\sum E P P$ discrepancy exists without significant changes in MEPP amplitudes. $\boldsymbol{A}$, Sample MEPPs recorded at times marked by arrows in $\boldsymbol{B}$. Calibration: $1 \mathrm{mV}, 1 \mathrm{~s}$. $\boldsymbol{B}$, MEPP frequency transiently increased during and after each train. Arrows mark times sampled in A. C, MEPP amplitudes during the entire experiment (moving bin average of 9 consecutive MEPPS). Trains were delivered at times marked by short bars. $D$, Average \pm SD amplitudes of MEPPs before first stimulus train, between each train, and after last train. $\boldsymbol{E}$, Total quanta released asynchronously and by nerve stimulation, and their sum. MEPPs were measured during and between trains. $\boldsymbol{F}$, Sample recordings taken at early (top trace), middle (middle trace), and late (lowest trace) times during train 6 (see arrows in $\boldsymbol{G}$ ). Evoked responses were $10 \mathrm{~ms}$ apart. The increased frequency of asynchronous release during the later stages is evident. $G$, EPPs recorded during trains 1,6 , and 9 . The arrows in the middle panel show the times at which recordings in $\boldsymbol{F}$ were taken. $\boldsymbol{H}$, Fluorescence changes during six consecutive, identical stimulus trains ( $100 \mathrm{~Hz}$ for $2 \mathrm{~s}$ ). The downward deflection at the start of each train is caused by a slight movement artifact. Fluorescence baseline for each train was measured from the preceding point. I, Fluorescence change per train (filled circles) remained nearly constant, whereas total quanta (large circles) progressively declined. a.u., Arbitrary units.

(ACh) release from partially filled synaptic vesicles and a reduction in muscle fiber input resistance as possible explanations. These conclusions are based on the assumption that evoked EPPs and asynchronous MEPPs are drawn from the same pop- ulation, a long-held assumption that was challenged (Sara et al., 2005) but recently reaffirmed (Groemer and Klingauf, 2007).

What, then, might explain the discrepant results between optical and electrical estimates of secretion? Here, we consider four possibilities. First, ectopic release of ACh (for example, from the nerve terminal surface that faces the Schwann cell) could generate a fluorescence signal without commensurate muscle depolarization. Although evidence of ectopic vesicle cycling has been reported (Rizzoli and Betz, 2004), the amount needed to explain the magnitude of the discrepancy (approximately one-half of the total release) that we observed is far greater than estimated to occur, making ectopic release of ACh an unlikely explanation. Second, we cannot rule out the possibility that intense stimulation somehow produces a transient dequenching of intracellular $\mathrm{spH}$ in vesicles or other structures that do not undergo exocytosis, thereby raising nerve terminal fluorescence without secreting ACh. The recovery time course of such a process would have to match that of endocytosis and requenching of $\mathrm{spH}$, to produce the overall monophasic recovery that we observed. Third, if $\mathrm{spH}$ expression in vesicles is heterogeneous and $\mathrm{spH}$ interferes with docking or exocytosis, then vesicles with fewer spH molecules (and therefore lower fluorescence during exocytosis) would be preferentially released, and vesicles coming later would be brighter. The effect would have to be large, however, to account fully for the observed discrepancy. Finally, evoked exocytosis of vesicles or other spH-bearing organelles that contain no ACh would produce the observed effect. Exocytosis of empty synaptic vesicles (Parsons et al., 1999) and secretory granules (Tabares et al., 2001; Croft et al., 2005) has been demonstrated, although at the synapse their appearance is preceded by exocytosis of partially filled vesicles, giving rise to MEPPs of reduced amplitude, which we did not observe. Other intracellular membrane-bound compartments might contain quenched $\mathrm{spH}$ but lack ACh transporters and might become fusogenic during the rise of intracellular calcium-ion concentration produced by intense stimulation. Such structures might include large dense-core granules or endosomes not in the usual synaptic vesicle recycling pathway.

\section{References}

Atluri PP, Ryan TA (2006) The kinetics of synaptic vesicle reacidification at hippocampal nerve terminals. J Neurosci 26:2313-2320. 
Bozza T, McGann JP, Mombaerts P, Wachowiak M (2004) In vivo imaging of neuronal activity by targeted expression of a genetically encoded probe in the mouse. Neuron 42:9-21.

Caroni P (1997) Overexpression of growth-associated proteins in the neurons of adult transgenic mice. J Neurosci Methods 71:3-9.

Croft BG, Fortin GD, Corera AT, Edwards RH, Beaudet A, Trudeau L-E, Fon EA (2005) Normal biogenesis and cycling of empty synaptic vesicles in dopamine neurons of vesicular monoamine transporter 2 knockout mice. Mol Biol Cell 16:306-315.

Fernandez-Alfonso T, Kwan R, Ryan TA (2006) Synaptic vesicles interchange their membrane proteins with a large surface reservoir during recycling. Neuron 51:179-186.

Gaffield MA, Rizzoli SO, Betz WJ (2006) Mobility of synaptic vesicles in different pools in resting and stimulated frog motor nerve terminals. Neuron 51:317-325.

Gandhi SP, Stevens CF (2003) Three modes of synaptic vesicular recycling revealed by single-vesicle imaging. Nature 423:607-613.

Granseth B, Odermatt B, Royle SJ, Lagnado L (2006) Clathrin-mediated endocytosis is the dominant mechanism of vesicle retrieval at hippocampal synapses. Neuron 51:773-786.

Groemer TW, Klingauf J (2007) Synaptic vesicles recycling spontaneously and during activity belong to the same vesicle pool. Nat Neurosci 10:145-147.

Han W, Rhee JS, Maximov A, Lin W, Hammer RE, Rosenmund C, Sudhof TC (2005) C-terminal ECFP fusion impairs synaptotagmin 1 function: crowding out synaptotagmin 1. J Biol Chem 280:5089-5100.

Harata N, Pyle JL, Aravanis AM, Mozhayeva M, Kavalali ET, Tsien RW (2001) Limited numbers of recycling vesicles in small CNS nerve terminals: implications for neural signaling and vesicular cycling. Trends Neurosci 11:637-643.

Harata NC, Choi S, Pyle JL, Aravanis AM, Tsien RW (2006) Frequencydependent kinetics and prevalence of kiss-and-run and reuse at hippocampal synapses studied with novel quenching methods. Neuron 49:243-256.

He L, Wu XS, Mohan R, Wu LG (2006) Two modes of fusion pore opening revealed by cell-attached recordings at a synapse. Nature 444:102-105.

Hennig R, Lomo T (1985) Firing patterns of motor units in normal rats. Nature 314:164-166.

Heuser JE, Reese TS (1973) Evidence for recycling of synaptic vesicle membrane during transmitter release at the frog neuromuscular junction. J Cell Biol 57:315-344.

Hogan B, Constantini F, Lacey E (1994) Manipulating the mouse embryo: a laboratory manual. Cold Spring Harbor, NY: Cold Spring Harbor Laboratory.

Katz E, Ferro PA, Weisz G, Uchitel OD (1996) Calcium channels involved in synaptic transmission at the mature and regenerating mouse neuromuscular junction. J Physiol 497:687-697.

Li Z, Burrone J, Tyler WJ, Hartman KN, Albeanu DF, Murthy VN (2005) Synaptic vesicle recycling studied in transgenic mice expressing synaptopHluorin. Proc Natl Acad Sci USA 102:6131-6136.

Linares-Clemente P, Garcia-Junco Clemente P, Pintado O, FernandezChacon R (2005) SynaptopHluorin transgenic mice to monitor synaptic vesicle fusion in single nerve terminals. J Physiol Biochem 61:163.

Miesenbock G, De Angelis DA, Rothman JE (1998) Visualizing secretion and synaptic transmission with $\mathrm{pH}$-sensitive green fluorescent proteins. Nature 394:192-195.

Ng M, Roorda RD, Lima SQ, Zemelman BV, Morcillo P, Miesenbock G
(2002) Transmission of olfactory information between three populations of neurons in the antennal lobe of the fly. Neuron 36:463-474.

Nishimune H, Sanes JR, Carlson SS (2004) A synaptic laminin-calcium channel interaction organizes active zones in motor nerve terminals. $\mathrm{Na}$ ture 432:580-587.

Parsons RL, Calupca MA, Merriam LA, Prior C (1999) Empty synaptic vesicles recycle and undergo exocytosis at vesamicol-treated motor nerve terminals. J Neurophysiol 81:2696-2700.

Prechtl JC, Cohen LB, Pesaran B, Mitra PP, Kleinfeld D (1997) Visual stimuli induce waves of electrical activity in turtle cortex. Proc Natl Acad Sci USA 94:7621-7626.

Probst A, Gotz J, Wiederhold KH, Tolnay M, Mistl C, Jaton AL, Hong M, Ishihara T, Lee VM, Trojanowski JQ, Jakes R, Crowther RA, Spillantini MG, Burki K, Goedert M (2000) Axonopathy and amyotrophy in mice transgenic for human four-repeat tau protein. Acta Neuropathol (Berl) 99:469-481.

Richards DA, Guatimosim C, Betz WJ (2000) Two endocytic recycling routes fill two vesicle pools in frog motor nerve terminals. Neuron 27:551-559.

Rizzoli SO, Betz WJ (2004) The structural organization of the readily releasable pool of synaptic vesicles. Science 303:2037-2039.

Sankaranarayanan S, Ryan TA (2000) Real-time measurements of vesicleSNARE recycling in synapses of the central nervous system. Nat Cell Biol 2:197-204.

Sankaranarayanan S, De Angelis D, Rothman JE, Ryan TA (2000) The use of pHluorins for optical measurements of presynaptic activity. Biophys J 79:2199-2208.

Sara Y, Virmani T, Deak F, Liu X, Kavalali ET (2005) An isolated pool of vesicles recycles at rest and drives spontaneous neurotransmission. Neuron 45:563-573.

Tabares L, Ales E, Lindau M, Alvarez de Toledo G (2001) Exocytosis of catecholamine (CA)-containing and CA-free granules in chromaffin cells. J Biol Chem 276:39974-39979.

Teng H, Cole JC, Roberts RL, Wilkinson RS (1999) Endocytic active zones: hot spots for endocytosis in vertebrate neuromuscular terminals. J Neurosci 19:4855-4866.

Vidal M, Morris R, Grosveld F, Spanopoulou E (1990) Tissue-specific control elements of the Thy-1 gene. EMBO J 9:833-840.

Voglmaier SM, Kam K, Yang H, Fortin DL, Hua Z, Nicoll RA, Edwards RH (2006) Distinct endocytic pathways control the rate and extent of synaptic vesicle protein recycling. Neuron 51:71-84.

Wienisch M, Klingauf J (2006) Vesicular proteins exocytosed and subsequently retrieved by compensatory endocytosis are nonidentical. Nat Neurosci 9:1019-1027.

Wu L-G, Betz WJ (1999) Spatial variability in release at the frog neuromuscular junction measured with FM1-43. Can J Physiol Pharmacol 77:672-678.

Wyatt RM, Balice-Gordon RJ (2005) Genetically encoded reporters of neurotransmitter release and terminal size at mouse neuromuscular and other synapses. Soc Neurosci Abstr 31:26.1.

Wyatt RM, Balice-Gordon RJ (2006) Synaptic vesicle dynamics at neuromuscular synapses of synapto-pHluorin expressing mice. Soc Neurosci Abstr 32:336.3.

Zefirov A, Benish T, Fatkullin N, Cheranov S, Khazipov R (1995) Localization of active zones. Nature 376:393-394.

Zenisek D, Steyer JA, Almers W (2000) Transport, capture and exocytosis of single synaptic vesicles at active zones. Nature 406:849-854. 